\title{
Paradoxical increase of exploratory behavior in the elevated plus-maze by rats exposed to two kinds of aversive stimuli
}

S. Morato and M.L. Brandão

\author{
Faculdade de Filosofia, Ciências e Letras de Ribeirão Preto \\ Universidade de São Paulo, Ribeirão Preto, SP, Brasil
}

\begin{abstract}
Correspondence
S. Morato

Faculdade de Filosofia, Ciências e Letras de Ribeirão Preto, USP Av. Bandeirantes, 3900 14040-901 Ribeirão Preto, SP Brasil

Fax: 55 (016) 633-5015

E-mail: silmorat@usp.br

Research supported by CNPq (No. 400702/93-2). Publication supported by FAPESP.
\end{abstract}

Received February 14, 1997 Accepted July 29, 1997

\begin{abstract}
Albino rats were submitted to a 24-h period of social isolation (individual housing) combined with $0,1,2$ or 3 twenty-four-hour periods of exposure to different vivaria (novelty) and tested in the elevated plus-maze. Results, reported as mean \pm SEM for $\mathrm{N}=12$, show that the time (in seconds) spent in the open arms by rats exposed to novelty for $0,1,2$ and 3 days was $28.3 \pm 4.4,31.6 \pm 3.2,29.1 \pm 3.5$ and $25.0 \pm 3.3$, respectively, when grouped in the same vivarium; 29.6 $\pm 2.7,7.6 \pm 2.1,9.6 \pm 4.4$ and $28.5 \pm 3.7$ when grouped in different vivaria; $2.9 \pm 1.1,1.8 \pm 1.0,2.7 \pm 1.1$ and $0 \pm 0$ when isolated in the same vivarium, and $2.6 \pm 1.1,31.5 \pm 8.2,24.8 \pm 4.2$ and $0 \pm 0$ when isolated in different vivaria. The number of entries into the open and closed arms followed a similar trend. This indicates that, separately, both exposure to novelty and isolation are aversive manipulations. Paradoxically, when novelty was combined with a concomitant 24-h period of social isolation prior to testing, the decrease in exploratory behavior caused by either of the two aversive manipulations alone was reverted. These results are indicative that less intense anxiety triggers mechanisms mediating less energetic behavior such as freezing, while higher levels trigger mechanisms mediating more vigorous action, such as flight/fight behavior, since the combination of two aversive situations resulted in more exploratory behavior than with either alone. They are also suggestive of habituation to the effects of novelty, since exposure to it for 3 days produced exploratory behavior similar to that of controls.
\end{abstract}

\section{Introduction}

The elevated plus-maze $(1,2)$, a modification of the procedure proposed by Montgomery (3), has often been used to investigate anxiolytic and anxiogenic compounds and to study the involvement of neurotransmitters in anxiety (e.g., 4-6). The method was considered
Key words

- Elevated plus-maze

- Social isolation

- Novelty

- Anxiety

- Exploratory behavior

- Aversive stimuli 
or end closing) and measuring the frequency of entries and time spent in each type of arm. A rat explores both the closed and the open arms but typically will enter more frequently and stay longer in the closed arms. The percent preference for open or closed arms, both for entries and duration $(2,5)$, is taken as an index of anxiety, with more intense anxiety being correlated with the lower percent preference for the open arms.

In spite of the apparent simplicity of this test situation, aversion to the open arms seems to be influenced by many factors such as sex (7), pre-exposure to the maze (8-10), raised edges in the open arms (10), type of floor (11), time of day at which testing occurs (9), and environmental levels of illumination $(9,11)$. We have shown that social isolation adds to the aversiveness of the open arms $(12,13)$. In those experiments, social isolation for different periods of time (2-24 h to 2 weeks) caused rats to explore the open arms less than controls, an effect that could be reversed or attenuated by drugs with an anxiolytic effect. Similar results had already been reported for rats isolated for six weeks and the findings have been associated with increased fearfulness manifested as decreased activity in the plus-maze (14). In addition, we have recently shown that the combination of two aversive manipulations (transporting the rats with a cart and social isolation) increases exploratory behavior in the open arms when compared to the effect of either cart transportation or social isolation alone, which only caused marked decreases in the exploration of the open arms (15).

In the present experiment we investigated the effects of combinations of novelty (exposure to different environments) and social isolation (individual housing) on the exploratory activity of rats in the plus-maze. We decided to investigate novelty because we noticed that previous exposure to different environmental stimuli altered the exploratory activity of rats submitted to the elevated plus-maze.

\section{Material and Methods}

\section{Animals}

Male Wistar rats (60-70 days) weighing 200-230 g were housed 6 to a plastic box (40 x 30 x $17 \mathrm{~cm}$ ) with food and water ad libitum on a 12-h light-dark cycle (lights on at 7:00 a.m.) for a habituation period of 3 days. According to the experimental protocol, the rats were then moved every $24 \mathrm{~h}$ to different vivaria with distinct environments (Table 1). Room temperature was maintained at 24$26^{\circ} \mathrm{C}$ in all vivaria. Twelve rats were studied under each condition. All testing was performed between 7:30 and 10:30 a.m.

\section{Apparatus}

Locomotor activity was measured just before the test in the maze in a wooden arena $(60 \times 60 \times 30 \mathrm{~cm}$ with the floor divided into $15-\mathrm{cm}$ squares) for $5 \mathrm{~min}$ because previous studies have shown that this procedure increases baseline exploratory activity in the plus-maze (2). Exploratory activity was then measured in an elevated plus-maze similar to that previously described by Pellow et al. (2).

Briefly, the maze was elevated $50 \mathrm{~cm}$ from the floor and consisted of two open arms (with 1-cm Plexiglas edges) and two closed arms (with 40-cm high wooden walls and no roof) arranged in such a way that like arms were opposite to each other.

The arena and the maze were located in a room lit by a $15-\mathrm{W}$ light bulb, $1.75 \mathrm{~m}$ above the central part of the maze. All data were recorded using standard Grason-Stadler (Concord, MA) electromechanical equipment in the test room.

\section{Experimental protocol}

Housing. The subjects were either kept in groups of six to a cage $(\mathrm{G})$ all the time or individually housed (I) in smaller cages (27 
$\mathrm{x} 15 \mathrm{x} 14 \mathrm{~cm}) 24 \mathrm{~h}$ prior to testing. The latter condition allowed them to see, hear and smell each other. All had free access to water and food throughout the experiment.

Novelty. Every $24 \mathrm{~h}$ the rats were exposed to novel environments, i.e., different vivaria with temperature control but differing in terms of room size, number of other experimental rats, noise of the ventilation systems, and the presence, size and location of windows. The subjects, in groups of 12 rats, were exposed to either the same $(\mathrm{S})$ or different vivaria (D) for $0,1,2$ or 3 days according to the experimental protocol (Table 1). The groups were coded $G$ or I, for grouped or individually housed, respectively, and S or $\mathrm{D}$, for same vivarium or different vivaria, plus a number indicating how many days the group was submitted to the novelty. Thus, group GD3 indicated the subjects grouped in different vivaria for 3 consecutive days, IS2 the subjects isolated in the same vivarium for 2 days, and so on.

Four different vivaria were used, arbitrarily denominated a, b, c and d. The characteristics of the vivaria were as follows. Vivarium a was $4.0 \times 2.0 \mathrm{~m}$ in size, had no windows or any other communication with the environment, with passive exhaust to another room where the air was actively pumped out, decreasing noise, with air conditioning directly installed in the room, and illumination provided by a fluorescent $40-\mathrm{W}$ lamp. This vivarium usually contained only the rats being tested. Vivarium $\mathrm{b}$ was $3.0 \mathrm{x}$ $2.0 \mathrm{~m}$ in size, had no windows but communicated to the outside through the exhaust fan hole which allowed air to be actively pumped out and illumination to enter. Insects were kept out with a fine wire mesh. Air conditioning was installed in the room, which was illuminated by a $20-\mathrm{W}$ fluorescent lamp. This vivarium usually contained about 10 other individually housed rats, usually recovering from brain surgery. Vivarium $\mathrm{c}$ was $5.0 \times 3.5 \mathrm{~m}$ in size, had a $3.5 \times 0.6-\mathrm{m}$ window in the upper part of one of the walls and an
Table 1 - Protocol of exposure to novelty for control and experimental rats.

Tests in the elevated plus-maze performed immediately after experimental manipulation.

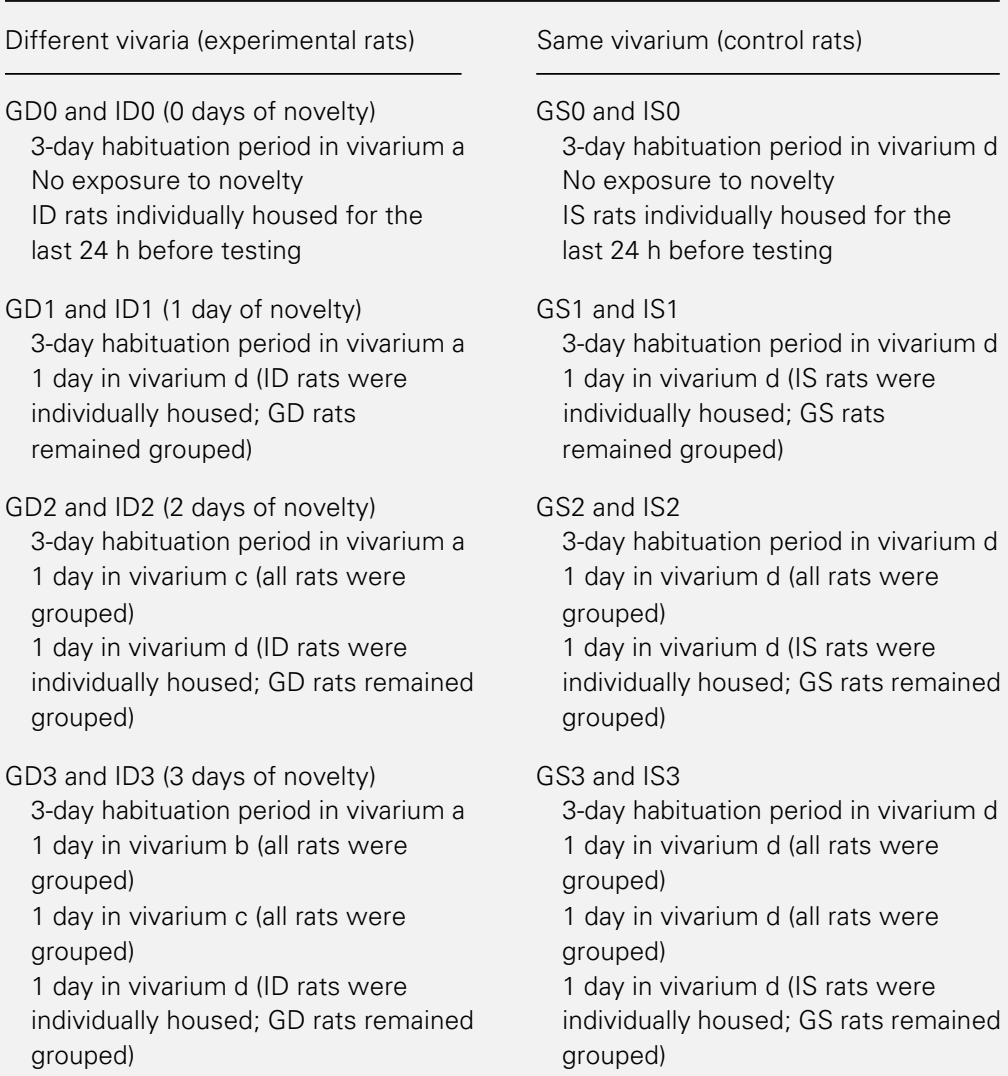

exhaust fan hole on an adjacent wall (which also permitted illumination in but kept insects out with a fine wire mesh). The air conditioner was installed in another room, decreasing noise level. The vivarium was illuminated by three $40-\mathrm{W}$ fluorescent lamps and usually contained an average of 50 resident rats. Vivarium d was $5.0 \times 3.5 \mathrm{~m}$ in size. One of the walls had a $1.0 \times 0.6-\mathrm{m}$ window (upper part), an exhaust fan hole (which also allowed illumination in but kept insects out with a fine wire mesh), and an air conditioner. It was illuminated by four $40-\mathrm{W}$ fluorescent lamps and usually held more than 100 resident rats.

All individual housing occurred during the last $24 \mathrm{~h}$ prior to testing. Rats belonging to groups GD0 and ID0 were habituated to 
vivarium a for three days and then tested. Likewise, GS0 and IS0 rats were habituated to vivarium $d$ for three days and then tested. GD1 and ID1 rats were habituated to vivarium a for three days, moved to vivarium $d$ for $24 \mathrm{~h}$ and then tested; GS1 and IS1 rats remained in vivarium $\mathrm{d}$ for the four days and then tested. The GD2 and ID2 groups were habituated to vivarium a for three days, moved to vivarium $\mathrm{c}$ for $24 \mathrm{~h}$, then moved to vivarium $\mathrm{d}$ for 24 additional hours and then tested; GS2 and IS2 animals remained in vivarium $\mathrm{d}$ for an equal length of time (five days) and were then tested. Finally, GD3 and ID3 rats were habituated to vivarium a for three days, spent three sequential 24-h periods in vivaria $\mathrm{b}, \mathrm{c}$ and $\mathrm{d}$, and were then tested; GS3 and IS3 rats remained an equal length of time in vivarium $d$ (six days) and were then tested.

Thus, except for the GD0 and ID0 subjects, that were only exposed to vivarium a and tested, GD and ID animals went through the vivaria in the same order: vivarium $b$ on the third day before testing, vivarium $\mathrm{c}$ on the second day before testing, and vivarium $\mathrm{d}$ on the day before testing. Also, again except for groups GD0 and ID0, vivarium d was the place where all social isolation occurred for both ID and IS animals.

Behavioral measurements. Each rat was placed individually in the arena for $5 \mathrm{~min}$ (where the number of squares crossed was counted) and then transferred to the elevated plus-maze where it was gently placed with the nose facing one of the closed arms. The number of entries and time spent in each type of arm was recorded for 5 min by an experimenter standing as still as possible and away from the maze. An entry was recorded when all four paws were placed inside one arm. The latency to the first entry was also recorded. This last measure, together with the number of squares crossed in the arena, was used to assess locomotor deficits, if any.

For analysis, the percent of the number of entries and time spent in the open arms were calculated in relation to total entries in both arms and total duration of the session, respectively.

\section{Statistical analysis}

The different degrees of exposure to novelty and the different housing conditions were analyzed separately using one way analysis of variance (ANOVA) and post hoc comparisons between groups, when necessary, using the Dunnett multiple comparisons test (16).

\section{Results}

\section{Total activity}

The general exploratory activity in the elevated plus-maze of rats submitted to the two housing conditions and to the different number of days of novelty exposure is shown in Figure 1 and Table 2. Figure 1 shows that novelty in the form of daily exposure to a different environment affected rats in the two housing conditions when compared to the respective 0-day exposed animals. Total entries by IS rats $\left(\mathrm{F}_{[3,44]}=4.204, \mathrm{P}<0.05\right)$ did not differ. Total entries were increased in GS1 rats $\left(\mathrm{F}_{[3,44]}=4.791, \mathrm{P}<0.01\right)$ and in ID1 and ID2 rats $\left(\mathrm{F}_{[3,44]}=22.505, \mathrm{P}<0.001\right)$, and decreased in GD1 and GD2 rats $\left(\mathrm{F}_{[3,44]}=\right.$ $8.755, \mathrm{P}<0.001$ ).

The effects of housing condition varied. When compared to GS animals, there was a decrease in total entries by IS0 and ID0 rats $\left(\mathrm{F}_{[3,44]}=72.099, \mathrm{P}<0.001\right)$ and IS3 and ID3 $\left(\mathrm{F}_{[3,44]}=68.324, \mathrm{P}<0.001\right)$. The rats in groups GD1 $\left(\mathrm{F}_{[3,44]}=13.411, \mathrm{P}<0.001\right)$ and GD2 $\left(\mathrm{F}_{[3,44]}=16.296, \mathrm{P}<0.001\right)$, but not ID1 and ID2, also showed decreased activity.

Table 2 shows that the number of crossings in the arena did not differ along novelty exposure days for $\mathrm{GS}\left(\mathrm{F}_{[3,44]}=1.583, \mathrm{P}>0.05\right)$, GD $\left(\mathrm{F}_{[3,44]}=2.516, \mathrm{P}>0.05\right)$, IS $\left(\mathrm{F}_{[3,44]}=\right.$ 
$0.970, \mathrm{P}>0.05)$ or ID rats $\left(\mathrm{F}_{[3,44]}=0.795\right.$, $\mathrm{P}>0.05)$. Neither was it different among housing conditions of $0\left(\mathrm{~F}_{[3,44]}=0.524, \mathrm{P}>0.05\right)$, $1\left(\mathrm{~F}_{[3,44]}=0.070, \mathrm{P}>0.05\right), 2\left(\mathrm{~F}_{[3,44]}=0.267\right.$, $\mathrm{P}>0.05)$ or $3\left(\mathrm{~F}_{[3,44]}=0.944, \mathrm{P}>0.05\right)$ days of exposure to novelty.

Table 2 also shows that the latency of first entry along novelty exposure days did not differ for $\mathrm{GS}\left(\mathrm{F}_{[3,44]}=0.681, \mathrm{P}>0.05\right)$ or ID rats $\left(\mathrm{F}_{[3,44]}=0.118, \mathrm{P}>0.05\right)$ but was slightly decreased for GD2 $\left(\mathrm{F}_{[3,44]}=3.219\right.$, $\mathrm{P}<0.05)$ and IS2 rats $\left(\mathrm{F}_{[3,44]}=4.417, \mathrm{P}<0.01\right)$. For housing conditions there were no differences for $0\left(\mathrm{~F}_{[3,44]}=1.266, \mathrm{P}>0.05\right), 1\left(\mathrm{~F}_{[3,44]}\right.$ $=0.169, \mathrm{P}>0.05), 2\left(\mathrm{~F}_{[3,44]}=0.165, \mathrm{P}>0.05\right)$ or $3\left(\mathrm{~F}_{[3,44]}=0.169, \mathrm{P}>0.05\right)$ days of exposure to novelty.

\section{Open arm exploration}

Figure 2 shows the frequency of entries into the open arms in terms of percentage of total entries. It can be seen that GS 1 animals displayed a decrease in the percent open arm entries $\left(\mathrm{F}_{[3,44]}=4.665, \mathrm{P}<0.01\right)$. However, there was an increase in closed arm entries $\left(\mathrm{F}_{[3,44]}=7.372, \mathrm{P}<0.001\right.$, data not shown $)$ by this group at the same time that no difference was observed for open arm entry frequency $\left(\mathrm{F}_{[3,44]}=0.613, \mathrm{P}>0.05\right.$, data not shown $)$. GD animals exhibited a decrease in percent open arm entries for 1- and 2-day exposed rats $\left(\mathrm{F}_{[3,44]}=15.885, \mathrm{P}<0.001\right)$. Novelty did not affect open arm $\left(\mathrm{F}_{[3,44]}=2.015, \mathrm{P}>0.05\right)$ entry percentage by IS rats but did cause increases in the percent of open arm entries $\left(\mathrm{F}_{[3,44]}=13.050, \mathrm{P}<0.001\right)$ by ID1 and ID2 animals.

In comparison to the corresponding GS groups, $0-\left(\mathrm{F}_{[3,44]}=21.361, \mathrm{P}<0.001\right)$ and 3 $\left(\mathrm{F}_{[3,44]}=308.120, \mathrm{P}<0.001\right)$ day exposed IS and ID animals and 1- $\left(\mathrm{F}_{[3,44]}=15.043\right.$, $\mathrm{P}<0.001)$ and $2-\left(\mathrm{F}_{[3,44]}=9.006, \mathrm{P}<0.001\right)$ day exposed GD and IS animals decreased the percent of open arm entries.

Figure 3 shows the time spent in the open arms. It can be seen that novelty did not
Table 2 - Number of square crossings in the arena and latency of the first entry.

Data are reported as mean \pm SEM. The groups are divided according to the number of days of exposure to novelty. ${ }^{*} P<0.05$ compared to the corresponding 0-day exposure group (Dunnett multiple comparisons test).

\begin{tabular}{lrl}
\hline Groups & \multicolumn{1}{c}{ Crossings } & Latency (s) \\
\hline Grouped in the same vivarium & \\
0 Days & $62.25 \pm 1.80$ & $5.75 \pm 0.45$ \\
1 Day & $69.75 \pm 2.36$ & $5.67 \pm 0.56$ \\
2 Days & $67.08 \pm 1.83$ & $4.92 \pm 0.29$ \\
3 Days & $63.58 \pm 2.34$ & $5.67 \pm 0.56$ \\
Grouped in different vivaria & \\
O Days & $62.17 \pm 1.65$ & $5.75 \pm 0.35$ \\
1 Day & $69.83 \pm 3.54$ & $4.92 \pm 0.26$ \\
2 Days & $68.00 \pm 1.85$ & $4.50 \pm 0.29^{*}$ \\
3 Days & $63.17 \pm 1.79$ & $4.92 \pm 0.26$ \\
Isolated in the same vivarium & \\
0 Days & $64.25 \pm 1.76$ & $5.50 \pm 0.31$ \\
1 Day & $69.50 \pm 2.69$ & $4.58 \pm 0.29$ \\
2 Days & $67.83 \pm 2.26$ & $3.75 \pm 0.45^{*}$ \\
3 Days & $67.42 \pm 2.10$ & $4.58 \pm 0.29$ \\
Isolated in different vivaria & \\
0 Days & $63.42 \pm 1.99$ & $4.92 \pm 0.26$ \\
1 Day & $68.25 \pm 2.38$ & $4.67 \pm 0.31$ \\
2 Days & $65.58 \pm 2.52$ & $4.83 \pm 0.52$ \\
3 Days & $65.83 \pm 1.93$ & $4.67 \pm 0.31$ \\
\end{tabular}

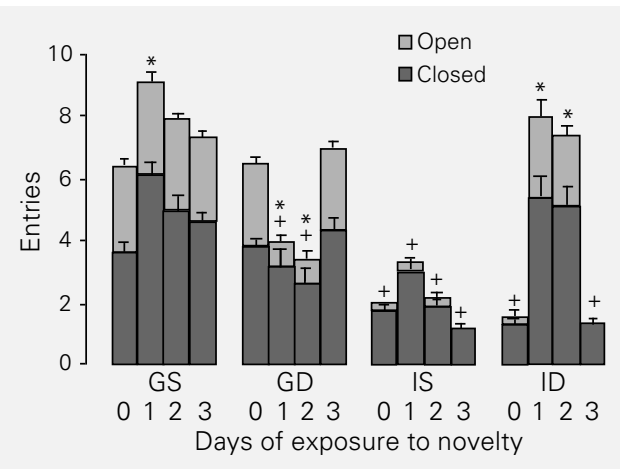

cause modifications in GS $\left(\mathrm{F}_{[3,44]}=0.556\right.$, $\mathrm{P}>0.05)$ or IS $\left(\mathrm{F}_{[3,44]}=2.049, \mathrm{P}>0.05\right)$ animals, while 1- and 2-day exposure reduced the time spent in the open arms by GD animals $\left(\mathrm{F}_{[3,44]}=12.723, \mathrm{P}<0.001\right)$ and increased the time spent in the open arms by ID animals $\left(\mathrm{F}_{[3,44]}=11.706, \mathrm{P}<0.001\right)$.

Compared to the corresponding GS
Figure 1 - Total number (mean \pm SEM) of entries into both arms. The rats were exposed to novelty for $0,1,2$ or 3 days. GS, Rats grouped in the same vivarium; $G D$, rats grouped in different vivaria; IS, rats isolated in the same vivarium, and ID, rats isolated in different vivaria. ${ }^{*} \mathrm{P}<0.05$ compared to the corresponding 0-day exposure group; $+P<0.05$ compared to the same novelty exposure GS group (Dunnett multiple comparisons test). 
Figure 2 - Percent (mean \pm SEM) of entries into the open arms. GS, Rats grouped in the same vivarium; GD, rats grouped in different vivaria; IS, rats isolated in the same vivarium; ID, rats isolated in different vivaria. ${ }^{*} \mathrm{P}<0.05$ compared to the corresponding 0 -day exposed group; ${ }^{+} \mathrm{P}<0.05$ compared to the corresponding GS group (Dunnett multiple comparisons test).

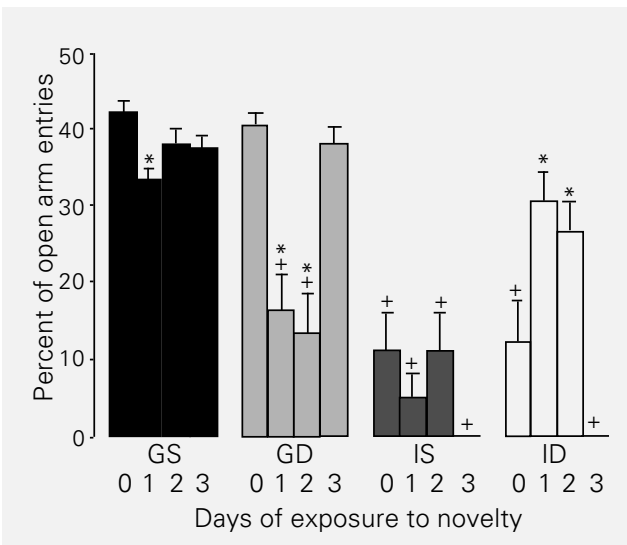

Figure 3 - Time (mean \pm SEM) spent in the open arms. GS, Rats grouped in the same vivarium; $G D$, rats grouped in different vivaria; IS, rats isolated in the same vivarium; ID, rats isolated in different vivaria. ${ }^{*} \mathrm{P}<0.05 \mathrm{com}-$ pared to the corresponding 0-day exposed group; $+\mathrm{P}<0.05$ compared to the corresponding GS group (Dunnett multiple comparisons test)

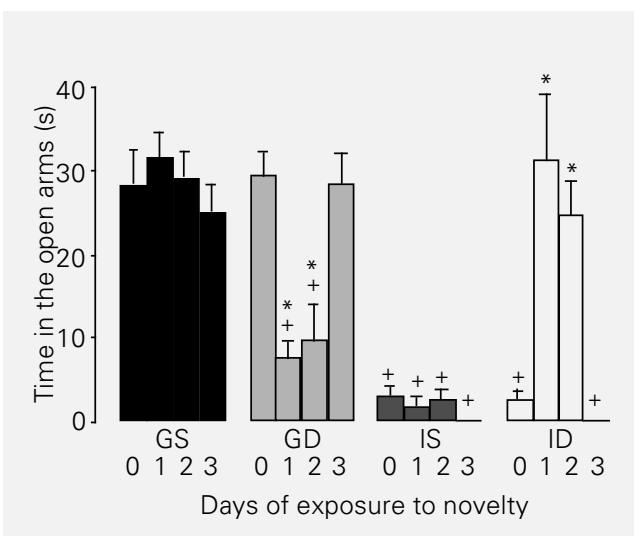

groups, IS0, ID0 $\left(\mathrm{F}_{[3,44]}=31.773, \mathrm{P}<0.001\right)$, IS3, ID3 $\left(\mathrm{F}_{[3,44]}=39.215, \mathrm{P}<0.001\right)$, GD1, IS1 $\left(\mathrm{F}_{[3,44]}=11.964, \mathrm{P}<0.001\right)$, and GD2 and IS2 $\left(\mathrm{F}_{[3,44]}=12.456, \mathrm{P}<0.001\right)$ rats exhibited a decrease in the time spent in the open arms.

\section{Discussion}

The effects of social isolation on the animals in the 0-day groups housed in the same vivarium or in different ones were similar and the subjects behaved in ways already described in the literature: grouped rats behaved similarly and explored about the same amount, whereas socially isolated rats showed a decrease in the exploration of the open arms (sometimes also decreasing closed arm entries) both in terms of number of entries and time (12-14). These results were independent of the rats being habituated to the vivarium adjacent to the test room or to any other vivarium located farther away.

The overall effect of novelty on the exploratory behavior of rats in the elevated plus-maze was to reverse the effects of housing conditions. Exposure to novelty for one or two days decreased total number of entries, percent of entries and time spent in the open arms by grouped animals while paradoxically increasing these parameters for socially isolated animals up to the control values of GS rats. Neither of these effects can be attributed to locomotor alterations judging by the number of square crossings in the arena and the latency of first entry which were not greatly altered.

Exposure to novelty in the form of a 24h stay in a new vivarium is most likely an aversive manipulation. This can be inferred from the decrease in exploratory behavior observed with the grouped animals, which was similar to the decrease caused by social isolation alone observed here and elsewhere $(12,13)$. What remains to be accounted for, then, is why the addition of two aversive manipulations (social isolation and novelty) restored exploratory behavior instead of reducing it even more. Either or both of the two hypotheses presented below may account for it.

First, it is plausible that low levels of aversion trigger behavioral mechanisms differing from those triggered by higher levels. Thus, as stated elsewhere (17-21), defensive behaviors are hierarchically organized and different behaviors within this class are provoked by aversive stimuli of different intensities. For example, freezing is the result of mild or distant threatening stimuli whereas flight/fight behaviors are the outcome of stronger or near stimuli. Likewise, in the present experiment, social isolation alone triggered mechanisms similar to those that mediate freezing while the combination of social isolation plus novelty may trigger other mechanisms more similar to those mediating more vigorous behavior such as fleeing or fighting. Consequently, the first case would 
make the animals less prone to exploring and the second case would make the animals more inclined to physical activity which, in the maze, would manifest itself as exploratory activity.

Consistent with this hypothesis, a recent study from this laboratory demonstrated that rats exposed to an elevated plus-maze with transparent walls, in which height is more prominent, showed higher exploratory activity than when exposed to the standard test in an elevated plus-maze with conventional wooden walls (22). Also, we have recently reported (15) that the combination of two different aversive stimuli (social isolation and transportation on a metal cart) caused an increase rather than a decrease in exploratory activity in the elevated plus-maze. As was the case in the present experiment, an aversive stimulus alone decreased measures related to open arm exploration but produced the opposite effect when combined with the other.

As a second hypothesis, these data may indicate two distinct states of anxiety generated by isolation and the novelty of different environments. Regarding novelty, another fact emerges from the data obtained. Expo- sure to novelty for three days seems to paradoxically bring the alterations back to the usual picture obtained with the control condition and the social isolation in the plusmaze. These behavioral effects seem to be related to phobic states different from the anxiety state induced by isolation. The phobic state is acquired and expressed during the first two exposures to the different environments and undergoes habituation or extinction during the third exposure. In fact, it has been reported that phobic states are extinguished by repeated testing (23). The anxiety state generated by isolation, on the contrary, tends to increase with time $(12,13,24)$. Thus, the animals might habituate to being constantly in the novelty situation and this would render them less reactive to it after a certain time, leaving only the effects of social isolation to be expressed.

Nevertheless, further experimentation is necessary in order to accept or reject these explanations, such as using anxiolytics to assess the aversiveness of novelty or manipulations that increase locomotor activity to investigate whether the effects observed here are really linked to anxiety or are of a more general nature.

\section{References}

1. Cruz APM, Frei F \& Graeff FG (1994). Ethopharmacological analysis of rat behavior on the elevated plus-maze. Pharmacology, Biochemistry and Behavior, 49: 171-176

2. Pellow $S$, Chopin $P$, File SE \& Briley $M$ (1985). Validation of open:closed arm entries in an elevated plus-maze as a measure of anxiety in the rat. Journal of Neuroscience Methods, 14: 149-167.

3. Montgomery KC (1955). The relation between fear induced by novel stimulation and exploratory behavior. Journal of Comparative and Physiological Psychology, 48: 254-260.

4. Critchley MAE \& Handley SL (1987). Effects in the X-maze anxiety model of agents acting at $5-\mathrm{HT}_{1}$ and $5-\mathrm{HT}_{2}$ receptors. Psychopharmacology, 93: 502-506.
5. Handley SL \& Mithani S (1984). Effects of alpha-adrenoceptor agonists and antagonists in a maze-exploration model of 'fear'-motivated behaviour. NaunynSchmiedeberg's Archives of Pharmacology, 327: 1-5.

6. Pellow S \& File SE (1986). Anxiolytic and anxiogenic drug effects on exploratory activity in an elevated plus-maze: a novel test of anxiety in the rat. Pharmacology, Biochemistry and Behavior, 24: 525-529.

7. Johnston AL \& File SE (1991). Sex differences in animal tests of anxiety. Physiology and Behavior, 49: 245-250.

8. File SE \& Zangrossi Jr H (1993). "One-trial tolerance" to the anxiolytic actions of benzodiazepines in the elevated plus-maze, or the development of a phobic state? Psychopharmacology, 110: 240-244.
9. Griebel G, Moreau JL, Jenk F, Martin JR \& Misslin R (1993). Some critical determinants of the behaviour of rats in the elevated plus-maze. Behavioural Processes, 29: 37-48

10. Treit D, Menard J \& Royan C (1993). Anxiogenic stimuli in the elevated plus-maze. Pharmacology, Biochemistry and Behavior, 44: 463-469.

11. Morato S \& Castrechini $P$ (1989). Effects of floor surface and environmental illumination on exploratory activity in the elevated plus-maze. Brazilian Journal of Medical and Biological Research, 22: 707710. 
12. Maisonnette $\mathrm{S}$, Morato $\mathrm{S} \&$ Brandão $\mathrm{ML}$ (1993). Role of resocialization and of 5$\mathrm{HT}_{1 \mathrm{~A}}$ receptor activation on the anxiogenic effects induced by isolation in the elevated plus-maze test. Physiology and Behavior, 54: 753-758.

13. Motta V, Maisonnette S, Morato S, Castrechini P \& Brandão ML (1992). Effects of blockade of $5-\mathrm{HT}_{2}$ receptors and activation of $5-\mathrm{HT}_{1} \mathrm{~A}$ receptors on the exploratory activity of rats in the elevated plus-maze. Psychopharmacology, 107: 135-139.

14. Jankowska E, Pucilowski O \& Kostowski W (1991). Chronic oral treatment with diltiazem or verapamil decreases isolationinduced activity impairment in elevated plus-maze. Behavioural Brain Research, 43: 155-158.

15. Morato S \& Brandão ML (1996). Transporting rats to the test situation on a cart can modify rat exploratory behavior in the elevated plus-maze. Psychobiology, 24: 247-252.
16. Bruning JL \& Kintz BL (1977). Computational Handbook of Statistics. Scott Foresman and Company, Glenview, Illinois.

17. Blanchard RJ \& Blanchard DC (1987). An ethoexperimental approach to the study of fear. Psychological Record, 37: 305316.

18. Blanchard RJ, Flannelly KJ \& Blanchard DC (1986). Defensive behaviors of laboratory and wild Rattus norvegicus. Journal of Comparative Psychology, 100: 101107.

19. Deakin JFW \& Graeff FG (1991). 5-HT and mechanisms of defence. Psychopharmacology, 5: 305-315.

20. Hediger H (1968). The Psychology of Animals in Zoos and Circuses. Dover, New York.

21. Ratner SC (1967). Comparative aspects of hypnosis. In: Gordon JE (Editor), Handbook of Clinical and Experimental Hypnosis. Macmillan, New York, 550-587.
22. Anseloni VZ, Messias G \& Brandão $M L$ (1995). Behavioral and pharmacological validation of the elevated plus maze constructed with transparent walls. Brazilian Journal of Medical and Biological Research, 28: 597-601.

23. File SE (1993). The interplay of learning and anxiety in the elevated plus maze. Behavioural Brain Research, 58: 199-202.

24. Wright IK, Upton N \& Marsden CA (1991). Resocialization of isolation-reared rats does not alter their anxiogenic profile on the elevated X-maze model of anxiety. Physiology and Behavior, 50: 1129-1132. 